\title{
Odontoclastic Resorptive Lesions in a Dog
}

\author{
Hiroto YOSHIKAWA ${ }^{1) *}$, Kazuhiro WATANABE ${ }^{2)}$ and Tsuyoshi OZAWA ${ }^{1)}$ \\ ${ }^{1)}$ Ozawa Animal Hospital, 4, Niban-Uji, Uji city, Kyoto, 611-0021, and ${ }^{2)}$ Laboratory of Veterinary Surgery, Faculty of Applied Biological \\ Sciences, Gifu University, 1-1 Yanagido, Gifu 501-1193, Japan
}

(Received 11 October 2006/Accepted 11 September 2007)

ABSTRACT. We found odontoclastic resorptive lesions on premolars and molars in a 4- year-old miniature dachshund. The teeth had been extracted because the dentin was resorbed. In some teeth, the roots had been replaced by hard tissue, and so we amputated the crowns and curetted roots and alveolar bone. Histopathological examination revealed that the dentin was resorbed by odontoclasts and was replaced with bony tissue. Ten months later we found resorptive lesions in other teeth, and we treated them along with the first treatment. At the time of writing, since this is the first report of a dog with the same lesion in other teeth after the first treatment, we hope to establish better treatment and prevention methods.

KEY WORDS: canine, neck lesion, odontoclastic resorptive lesions.

Odontoclastic Resorptive Lesions (ORL) is a dental disease which often occurs in middle-aged and older cats. In cats, Odontoclasts which are activated by gingival inflammation resorb the dentin and the lesion is replaced by bony tissue [11]. The resorption begins in the cementum below the gingival margin [11]. In advanced cases, spontaneous fracture of the tooth crown often occurs [11]. Usually affected cats are asymptomatic, but if the inflammation develops into pulp tissue, the cats may develop severe oral pain [11]. Many researchers suggest that the influencing factors may be the gingivitis, foods, mechanical trauma, FIV/FeLV infection and an imbalance of calcium regulating hormones and glucocorticoid, but the exact cause has not been confirmed $[10,11]$. On the other hand, the pathoetiology of canine ORL is regarded as similar to that of feline ORL [5] and according to one report, root resorption was seen in $17.9 \%$ of randomly selected dogs[6] ,but there are not many reports $[1,4,8,11]$. In this instance, we treated and evaluated histopathologically a case which we diagnosed as ORL. A summary of this case follows.

This case was a 4-year-old male miniature dachshund, weighing $5.4 \mathrm{~kg}$. This dog was presented to our hospital to undergo castration and dental cleaning.

At the first presentation, the dog was in good general condition and had mild to moderate tartar accumulation and gingivitis. Under general anesthesia, after our routine oral cleaning, we noticed gingival bleeding, redness and swelling in bilateral mandibular third premolars (Fig. 1). There was no retraction of the gingiva. From these findings, we suspected external resorption. Later we examined the dog radiographically and it revealed radiolucent areas around the cervical area of the bilateral mandibular third premolars and first molars. In these teeth, the resorption had reached to the

\footnotetext{
* Correspondence to: Yoshikawa, H., Small Animal Clinical Sciences, Veterinary Teaching Hospital, Western College of Veterinary Medicine, University of Saskatchewan, Saskatoon, SK, S7N5B4, Canada.

e-mail: hiroto.yoshikawa@usask.ca
}

pulp (Fig. 2). Root apex lesions were also suspected in the bilateral mandibular first molars due to the radiolucent area of the apex. Resorption of the alveolar bone was not observed. Based on this, we treated the teeth surgically 205 days after the first presentation. Before the treatment decision, we performed an oral examination again. We found that the crowns of the bilateral mandibular first molars were missed (Fig. 3). Resorption had advanced and crowns had been lost on the bilateral mandibular third premolars. Moreover, resorption of the cervical area was found on the right mandibular second premolar, left mandibular second and fourth premolar and right maxilla first molar. Since the crowns were fragile, we amputated the crowns, curetted roots and alveolar bone using surgical bar. Then we inserted antibiotics (Oxytetracycline $\mathrm{HCl}$ ) into the roots and closed the roots using absorbable surgical sutures. In the teeth with dentin-bone ankylosis, we amputated the crowns, flattened the bone border and sutured (Fig. 4). The dog was adminis-

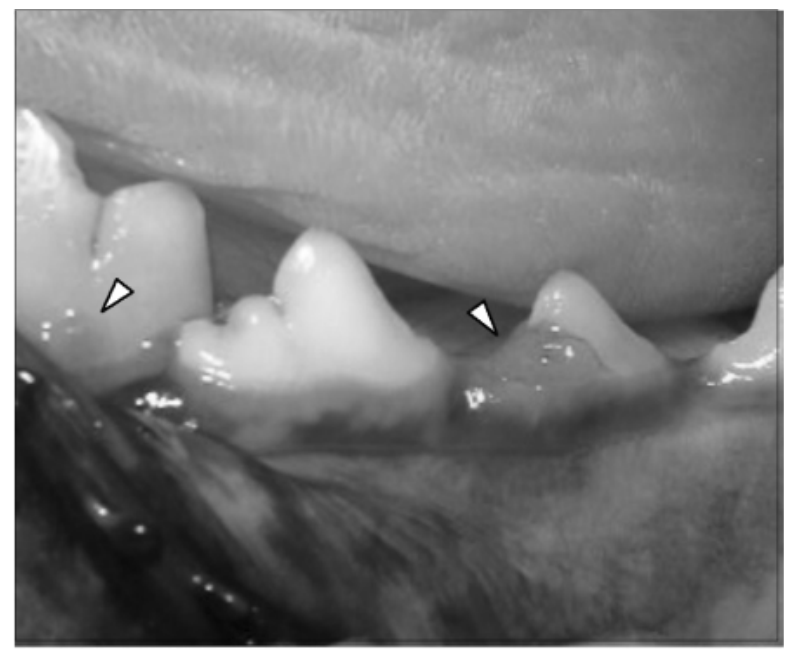

Fig. 1. Right mandibular third premolar and first molar at first presentation. Arrow heads indicate swelling of gingiva. 


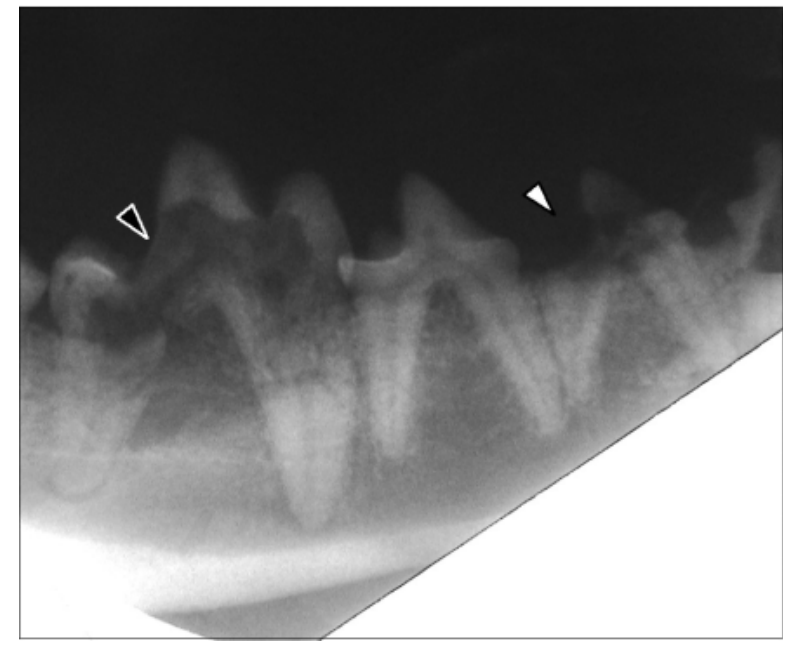

Fig. 2. X-ray film of right mandibular at day 20. Dentin of right mandibular third premolar (white arrow head) and first molar (black arrow head) is resorbed to the pulp.

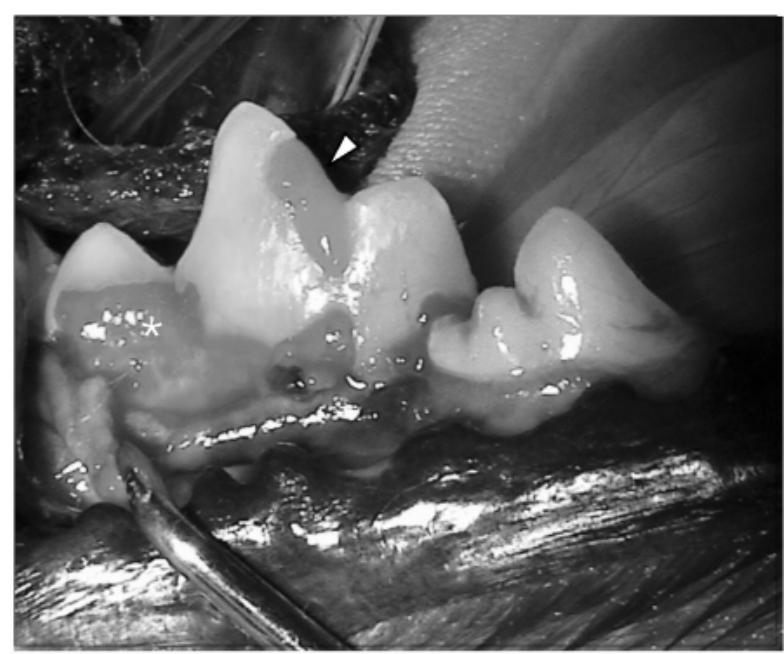

Fig. 3. Right mandibular first molar at day 205. Gingiva on the buccal side swelled and covered crown $\left(^{*}\right)$. Gingiva also proliferated from the tongue side to reach the buccal side (white arrow head).

tered clindamycin (12 mg/kg twice daily per os) for 14 days postoperatively.

At the follow-up physical examination 10 months later, the dog had new lesions on the right mandibular fourth premolar (Fig. 5), the bilateral mandibular second molars, the left maxilla second and fourth premolars and the left first and second molars which had all been intact at the first treatment. We then examined along with the last time and amputated the crowns.

We examined histopathologically all teeth removed. There was a heavy infiltration of lymphocyts and plasmacyts and there were increases of macrophages and fibroblasts in gingival submucosa of the right mandibular first

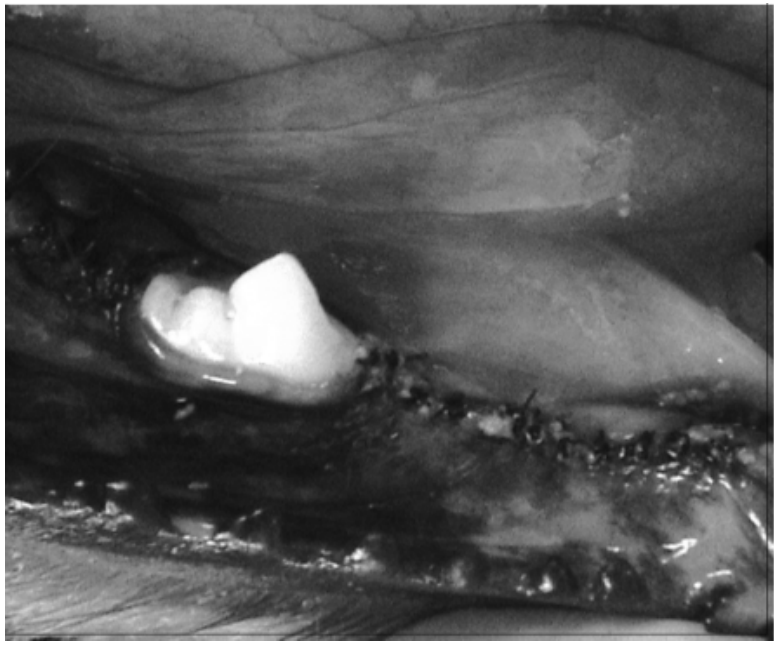

Fig. 4. Postoperative view of right mandibular at day 205. Fourth premolar was preserved since it appeared intact even under radiographic examination.

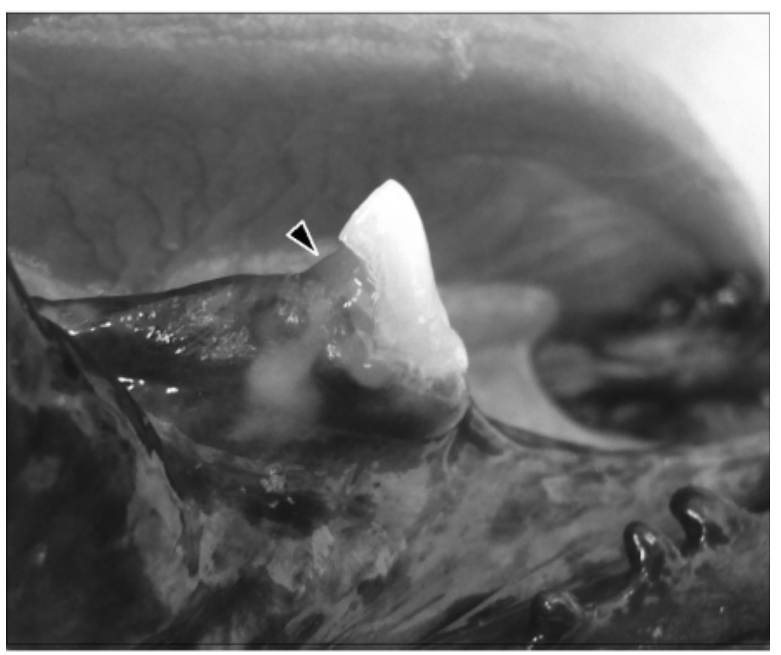

Fig. 5. Right mandibular fourth premolar is severely resorbed ten months after the first operation (arrow head).

molar, confirming gingivitis (Fig. 6). Dentins of the cervical area were severely and porously resorbed from the gingival mucosa side. Resorbed lesions had been replaced by boney tissue. These changes were similar to the ORL in cats [7]. There was no evidence of caries.

In cats, periodontal disease, mechanical trauma, abnormal occlusion and retrovirus infection seem to be the cause of ORL, but it has not yet been proven $[10,11]$. The dog we treated didn't have abnormal occlusion and radiographically there was no evidence of concurrent periodontal disease. Cats who gulp their food have a higher prevalence of ORL than cats who chew their food [11], but as of now, the relationship between occlusion and pathogenesis of ORL in dogs is not known. In cats, hypervitaminosis (especially 


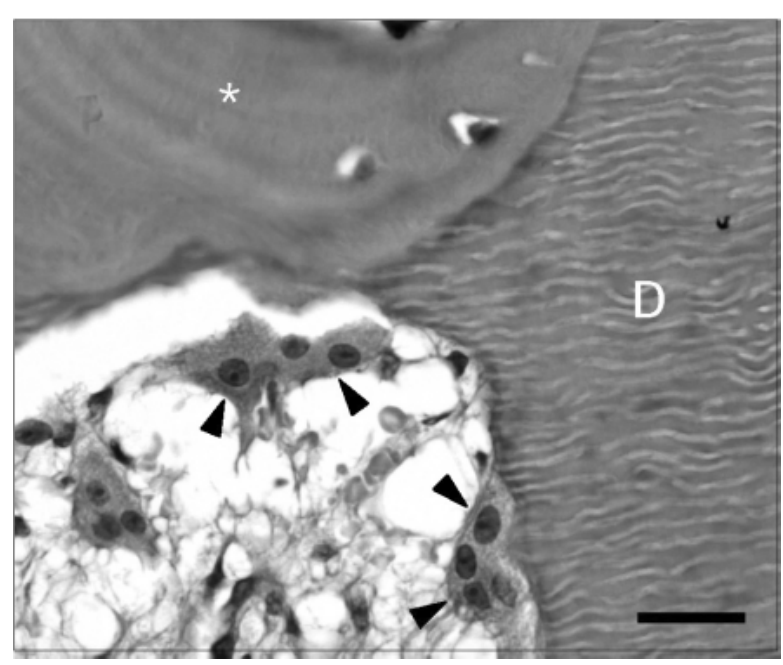

Fig. 6. Histopathological examination of right mandibular first molar. Dentin (D) was resorbed by odontoclasts (arrow head) and replaced by osteodentin $(*)$. H\&E stain. Bar $=25 \mu \mathrm{m}$.

vitamin A and D) may influence the activities of the osteoclast [10]. But this dog was fed named commercial food and sometimes fed treats, and didn't have a favorite toy. Dogs' teeth have a higher microhardness and higher magnesium content, whereas cats' teeth have higher calcium content [3, 11], and cats who vomit frequently have a higher prevalence of ORL [11]. Considering these points, more research is needed which compares teeth composition and the influence of vomit of affected dogs with those of non-affected dogs.

Because the symptoms (ie, oral pain and hypersalivation) may decrease cats' QOL, the objective of treating their ORL is to relieve them from oral pain $[2,9,11]$. There are some choices for treating ORL (ie, restoration, YAG laser, Extraction). But because all affected teeth in this dog were in an advanced stage when we treated, then, extraction was the only curative treatment. However, extraction often becomes much more difficult if ankylosis develops [9]. In that case, crown amputation is used if the cat doesn't have periodontal disease, radiographic evidence of endodontic disease or periapical pathologic findings, clinical evidence of stomatitis and positive results for FIV and FeLV [11]. In the dog we treated, ankylosis obstructed a routine extraction in some teeth, so we amputated crowns, and in some teeth where radiographically periapical pathologic disease was suspected, we curetted roots and alveolar bone. This time, crown amputation and suturing the gingiva was believed to be an effective choice of treatment, as is true in cats. In cats, ORL most commonly occurs in premolars and molars [9]. This dog had many more ORL-affected teeth than the proportion reported for cats [12] and had new lesions in some teeth which first were diagnosed as normal. This suggests that the etiology of ORL in dogs is different than in cats. At the time of writing, there are no reports of ORL development in turn in a dog. Consequently, there is also no established treatment and prevention.

Although ORL in dogs is relatively rare and is sometimes found by chance, it is important to diagnose radiographically at an early stage. Furthermore, since a recurrence of ORL in other teeth was observed in this dog and we have less knowledge about ORL than for cats, we hope that better treatment and prevention practices can be established through multi-faceted research.

\section{REFERENCES}

1. Bergstrom, A. 1992. Br. Vet. Dent. Assoc. J. 5: 5.

2. Burrows, C. F., Miller, W. H. and Harvey, C. E. 1987. pp. 34. In: Vet. Dent. (Harvey, C. E. ed.), W. B. Saunders, Philadelphia, U. S. A.

3. Crossley, D. A. 1995. J. Vet. Dent. 12: 111-113.

4. Eikenberg, S., Loheide, H. and Arens, F. C. 1998. J. Vet. Dent. 15: $175-178$.

5. Gorrel, C. 1998. Vet. Clin. North Am. Small Anim. Pract. 28: 1089-1110.

6. Hamp, S.E., Olsson, S.E. and Farso-Madsen, K. 1984. Vet. Radiol. Ultrasound. 25: 86-92.

7. Harvey, C. E. 1990. pp. 49-54. In: Manual of Small Anim. Dent. (Harvey, C. E. and Orr, H. S. eds.), British Small Animal Veterinary Association, Gloucestershire, U. K.

8. Kaplan, B. 1967. J. Am. Vet. Med. Assoc. 151: 708-709.

9. Lyon, K. F. 1992. Vet. Clin. North Am. Small Anim. Pract. 22: 1417-1432.

10. Okuda, A. and Harvey, C. E. 1992. Vet. Clin. North Am. Small Anim. Pract. 22: 1385-1404.

11. Reiter, A. M. and Mendoza, K. A. 2002. Vet. Clin. North Am. Small Anim. Pract. 32: 791- 837.

12. Wessum, R. van, Harvey, C. E. and Hennet, P. 1992. Vet. Clin. North Am. Small Anim. Pract. 22: 1405-1416. 